\title{
Incarcerated umbilical hernia with small bowel obstruction in a continuous ambulatory peritoneal dialysis patient
}

\author{
Yo-han Jeong", Jun-young Do', Mun-ju Hwang', Min-Jung Kim¹, Min Geun Gu', \\ Byung-sam Park', Jung-eun $\mathrm{Choi}^{2}$, Tae-woo Kim ${ }^{3}$ \\ Departments of ${ }^{1}$ Internal Medicine and ${ }^{2}$ General Surgery, College of Medicine, Yeungnam University, Daegu; ${ }^{3}$ Department of Internal \\ Medicine, Soonchunhayng University Gumi Hospital, Gumi, Korea
}

Patients treated with peritoneal dialysis have increased intra-abdominal pressure and a high prevalence of abdominal wall complications. Hernias can lead to significant morbidity in patients on peritoneal dialysis. Hernias are clinically important because of the risk of incarceration, strangulation and subsequent bowel obstruction, rupture, and peritonitis. In this paper, a case of incarcerated umbilical hernia with small bowel obstruction in a continuous ambulatory peritoneal dialysis (CAPD) patient is reported. The small bowel obstruction improved after herniorrhaphy, and the peritoneal dialysis was resumed 2 weeks after the herniorrhaphy. The patient had been undergoing CAPD without technical failure until the 2 months follow-up after the herniorrhaphy. This case shows that early detection of incarcerated umbilical hernia and herniorrhaphy can prevent resection of a strangulated small bowel so that it can remain on CAPD without post-operative technical failure. Umbilical hernias should be carefully observed and intestinal obstruction should be considered when a CAPD patient with an umbilical hernia has abdominal pain.

Keywords: Umbilical hernia; Intestinal obstruction; Peritoneal dialysis

\section{INTRODUCTION}

Patients undergoing peritoneal dialysis have increased intraabdominal pressure and a high prevalence of abdominal wall complications [1]. Hernias can lead to significant morbidity in patients on peritoneal dialysis. Hernias are clinically important because of the risk of incarceration, strangulation, subsequent bowel obstruction, rupture, and peritonitis. Those complications may also result in the failure of peritoneal dialysis as a dialysis modality due to damage of the peritoneal membrane [2].

In this paper, the authors report that early detection and herniorrhaphy of an incarcerated umbilical hernia with small bowel obstruction in a continuous ambulatory peritoneal dia-

Received: July 14, 2013; Revised: August 19, 2013;

Accepted: August 26, 2013

Corresponding Author: Jun-young Do, Department of Internal Medicine, College of Medicine, Yeungnam University,

170 Hyeonchung-ro, Namgu, Daegu 705-703, Korea

Tel: +82-53-680-3844, Fax: +82-53-654-8386

E-mail: jydo@med.yu.ac.kr lysis (CAPD) patient could lead to successful CAPD maintenance after an operation.

\section{CASE}

A 57-year-old female patient who had undergone CAPD 3 years earlier presented a bulging umbilical mass with whole abdominal pain and vomiting, which had been developing for 5 days. She was diagnosed with hypertension 7 years earlier. She had 1 episode of CAPD peritonitis with methicillin-resistant Staphylococcus aureus a year earlier.

Although she had this bulging painless intermittent umbilical mass for a year, the authors did not consider performing herniorrhaphy. Her whole abdominal pain intensified, and this tender bulging mass could not be manually reduced in the abdomen. Her blood pressure was $160 / 90 \mathrm{~mm} \mathrm{Hg}$ and body temperature was $37.3^{\circ} \mathrm{C}$. The authors performed peritoneal dialysate fluid analysis to confirm if her ailment was CAPD peritonitis. White blood cell count in the drained dialysate was $0 / \mu \mathrm{L}$, and red blood cell count $20 / \mu \mathrm{L}$. In complete 
blood count, white blood cell count was 6,970/ $\mu \mathrm{L}$, hemoglobin count $9.3 \mathrm{~g} / \mathrm{dL}$, and platelet count $227,000 / \mu \mathrm{L}$. Her serum blood urea nitrogen was $63.82 \mathrm{mg} / \mathrm{dL}$, and serum creatinine $8.6 \mathrm{mg} / \mathrm{dL}$. Suspecting bowel obstruction, the authors performed simple abdomen radiography and discovered much gas in the small bowel. Subsequently, abdominal computed tomography was performed, which revealed incarcerated umbilical hernia with small bowel obstruction (Fig. 1).

The authors decided to treat her hernia with surgery. Herniorrhaphy with Prolene Hernia System (PHS) mesh insertion was performed to repair her umbilical hernia. During the operation, an obstructed small bowel with a $22 \times 2 \mathrm{~cm}$ umbilical hernia was observed (Fig. 2).

After the operation, the patient was put on a fast for 7 days. Acute hemodialysis was conducted 3 times in three weeks until the small bowel obstruction improved, and her CAPD catheter was irrigated once daily with a $1.5 \%$ glucosebased dialysis solution to prevent its obstruction. After the herniorrhaphy, the abdominal pain decreased and the simple supine abdomen radiography showed an improvement in the dilated small bowel. The patient had fever during the postoperation period due to pneumonia in her left lower lobe, but she recovered with intravenous antibiotics treatment. Her peritoneal dialysis was resumed with a low exchange volume 2 weeks after her herniorrhaphy. The dialysate was drained well without abdominal pain. She underwent CAPD without technical failure during the 6 month follow-up after her herniorrhaphy.

\section{DISCUSSION}

Many complications have been described in patients undergoing peritoneal dialysis, such as infections (peritonitis and exit-site infection), complications associated with increased abdominal pressure (hernias, genital swelling, and hydrothorax), and mechanical problems with the catheter (obstruction and leakage) [3].

Hernia is considered one of the important complications of peritoneal dialysis. Its pathophysiology is based on the effect of increased abdominal pressure on the already weak abdominal wall [3]. Peritoneal dialysis patients have increased intra-peritoneal pressure due to the presence of the fill volume in the peritoneal cavity. The intra-abdominal pressure is proportional to the volume infused $[1,4,5]$. A large volume of
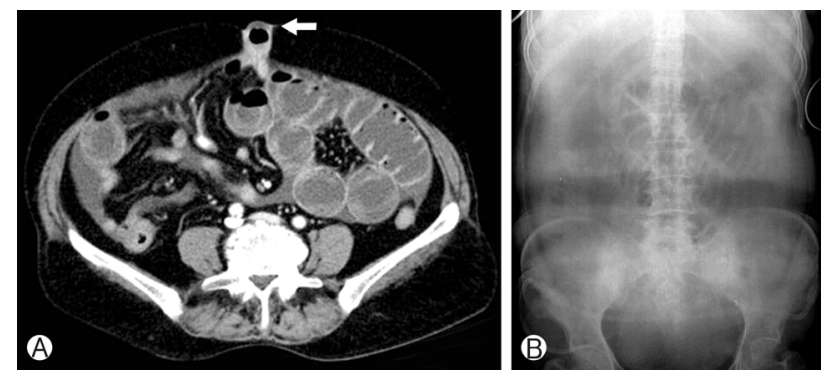

Fig. 1. (A) Abdominal computed tomography that shows incarcerated umbilical hernia with marked dilatation of the small bowel and a multiple air-fluid level (arrow) without large bowel gas. (B) Simple abdomen radiography that shows marked dilatation of the small bowel without large bowel gas.

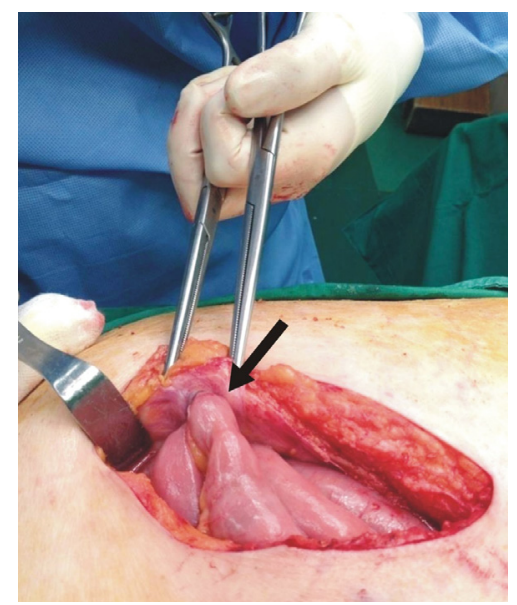

Fig. 2. Small bowel obstruction due to incarcerated umbilical hernia (arrow) that was observed during the herniorrhaphy.

dialysate is prescribed to improve the peritoneal small molecule clearance in order to make the clinical target level adequate [1]. Hernia has been found to be more prevalent among peritoneal dialysis patients than in the general population, with its reported frequencies of $10 \%$ to $25 \%$ and with an incidence of 0.06 hernias per dialysis year [3,6-8]. A few studies have reported that pre-existing hernias were detected in $5.5 \%$ to $12.3 \%$ of peritoneal dialysis patients before CAPD was initiated on the patient [1,3,9]. Most of the reported hernias can be differentiated as incisional in the area of the peritoneal catheter or umbilical and inguinal according to their locations [10].

Complications of hernia such as strangulation and rupture may also result in the failure of peritoneal dialysis as a dialysis modality due to damage of the peritoneal membrane [2]. It was reported that incarcerated hernias in peritoneal dialysis patients had the greatest likelihood of strangulation $[2,3]$. If 
the hernia is incarcerated or strangulated, abdominal catastrophes such as peritonitis secondary to strangulated hernia from CAPD peritonitis must be ruled out because their clinical manifestations are similar to those of CAPD peritonitis [11].

Therefore, abdominal wall hernias should be detected early to minimize mechanical complications [2]. The traditional operation method for abdominal wall hernia repair in peritoneal dialysis patients requires discontinuing the peritoneal dialysis for a long period of days to weeks after the operation to prevent damage of the repair site [12]. Polypropylene mesh repair was recently used for hernias of patients on CAPD $[3,7,13]$. Low-tension mesh repair provides a technically simple solution to the many surgical disadvantages of CAPD patients [13]. The method permits practically uninterrupted peritoneal dialysis without additional risk of hernia relapse [10]. Mesh hernia repair failure is generally rare and involved in unusual co-morbidity [14]. Prosthetic mesh repair of hernias in CAPD patients with vascular access problems seems to be useful for keeping such patients on this renal replacement therapy [7]. Most hernias are asymptomatic. Thus, they can be missed if possible vulnerable areas of the anterolateral abdominal wall are not carefully examined [3].

To prevent abdominal complications in peritoneal dialysis, hidden hernias must be evaluated carefully before peritoneal dialysis is initiated, and must be repaired during the peritoneal catheter insertion operation [1]. Addtionally, to prevent their recurrence, the use of the automated peritoneal dialysis method with low daytime exchange volumes may be considered for patients with a known abdominal wall problem [1].

This case illustrates that early detection of incarcerated umbilical hernia and herniorrhaphy can prevent the resection of a strangulated small bowel so that the patient can remain on CAPD without post-operative technical failure. An umbilical hernia in a CAPD patient should be carefully observed, and intestinal obstruction should be considered when a CAPD patient with a hernia complains of abdominal pain.

\section{CONFLICT OF INTEREST}

The authors have no conflict of interest.

\section{REFERENCES}

1. Del Peso G, Bajo MA, Costero O, Hevia C, Gil F, Díaz C, et al. Risk factors for abdominal wall complications in peritoneal dialysis patients. Perit Dial Int 2003;23:249-54.

2. Cherney DZ, Siccion Z, Chu M, Bargman JM. Natural history and outcome of incarcerated abdominal hernias in peritoneal dialysis patients. Adv Perit Dial 2004;20:86-9.

3. García-Ureña MA, Rodríguez CR, Vega Ruiz V, Carnero Hernández FJ, Fernández-Ruiz E, Vazquez Gallego JM, et al. Prevalence and management of hernias in peritoneal dialysis patients. Perit Dial Int 2006;26:198-202.

4. Gotloib L, Mines M, Garmizo L, Varka I. Hemodynamic effects of increasing intra-abdominal pressure in peritoneal dialysis. Perit Dial Bull 1981;1:41-3.

5. Twardowski ZJ, Prowant BF, Nolph KD, Martinez AJ, Lampton LM. High volume, low frequency continuous ambulatory peritoneal dialysis. Kidney Int 1983;23:64-70.

6. Bargman JM. Complications of peritoneal dialysis related to increased intraabdominal pressure. Kidney Int Suppl 1993; 40:S75-80.

7. Imvrios G, Tsakiris D, Gakis D, Takoudas D, Koukoudis P, Papadimitriou M, et al. Prosthetic mesh repair of multiple recurrent and large abdominal hernias in continuous ambulatory peritoneal dialysis patients. Perit Dial Int 1994;14:338-43.

8. Engeset J, Youngson GG. Ambulatory peritoneal dialysis and hernia complications. Surg Clin North Am 1984;64:385-92.

9. Afthentopoulos IE, Panduranga Rao S, Mathews R, Oreopoulos DG. Hernia development in CAPD patients and the effect of 2.51 dialysate volume in selected patients. Clin Nephrol 1998;49:251-7.

10. Ham JY, Shim DS, Kim KH, Kim HM, Kim SW, Lee OJ, et al. The Left Indirect Inguinal Hernia Associated with a Hydrocele on a CAPD Patient. Korean J Med 1994;46:735-8. Korean.

11. Lee BS, Park HK, Ju HW, Choi W, Kim SH, Park WD, et al. Strangulated umbilical hernia misdiagnosed as CAPD peritonitis. Korean J Nephrol 2007;26:641-5. Korean.

12. Pauls DG, Basinger BB, Shield CF 3rd. Inguinal herniorrhaphy in the continuous ambulatory peritoneal dialysis patient. Am J Kidney Dis 1992;20:497-9.

13. Lewis DM, Bingham C, Beaman M, Nicholls AJ, Riad HN. Polypropylene mesh hernia repair--an alternative permitting rapid return to peritoneal dialysis. Nephrol Dial Transplant 1998; 13:2488-9.

14. Abraham G, Nallathambi MN, Bhaskaran S, Srinivasan L. Recurrence of abdominal wall hernias due to failure of mesh repair in a peritoneal dialysis patient. Perit Dial Int 1997;17: 89-91. 